\title{
EL DIAGNÓSTICO DEL APRENDIZAJE DE LOS CONCEPTOS. UNA NECESIDAD EN LA DIRECCIÓN DEL PROCESO DE ENSEÑANZA APRENDIZAJE
}

The diagnosis of the learning of the concepts. a necessity in the address of the process of teaching learning

A diagnose da aprendizagem dos conceitos. uma necessidade no endereço do processo de ensinar aprendizagem

\section{Caridad Hernández Pérez}

Universidad de Ciencias Pedagógicas “Enrique José Varona” La Habana, Cuba. Fono: +5372609277 Correo electrónico: caridadhp@ucpejv.rimed.cu

\section{Resumen}

El diagnóstico del aprendizaje de los conceptos aporta indicadores de un alto valor teórico y metodológico para la dirección de la actividad pedagógica por el docente. Constituyen recursos metodológicos basados en la identificación de los conceptos y las relaciones semánticas entre estos, sus características, las cuales reflejan las vivencias, experiencias, las características de esos conocimientos adquiridos, las potencialidades, sin embargo en la práctica se observan carencias teóricas y metodológicas. Se deduce la necesidad de preparar al maestro en las exigencias teóricas y metodológicas para dirigir el aprendizaje de los conceptos de sus alumnos en su diversidad y crear condiciones para nuevos aprendizajes.

Palabras clave: aprendizaje, conceptos, recurso.

\begin{abstract}
The diagnosis of learning the concepts provides indicators of a high theoretical and methodological value for the direction of teaching activities by the teacher. Are methodological resources based on the identification of concepts and semantic relationships among these characteristics, which reflect the experiences, experiences, characteristics of these acquired knowledge, potential, however in practice theoretical and methodological deficiencies observed. The need to prepare the master in the theoretical
\end{abstract}


and methodological requirements to drive learning the concepts of their students in their diversity and create conditions for new learning follows.

Keywords: diagnosis; learning; concepts; resources methodological.

\section{Resumo}

O diagnóstico de aprender os conceitos fornece indicadores de um valor teórico e metodológico alto para a direção das atividades de ensino pelo professor. São recursos metodológicos com base na identificação de conceitos e relações semânticas entre essas características, que refletem as experiências, experiências, características destes conhecimentos adquiridos, potencial, no entanto, na prática, teórica e deficiências metodológicas observadas. a necessidade de preparar o mestre nos requisitos teóricos e metodológicos para conduzir a aprendizagem dos conceitos de seus alunos na sua diversidade e criar condições para um novo aprendizado segue.

Palavras chave: diagnostico; aprender; conceitos; recursos metodológicos.

\section{Introducción}

La Educación constituye uno de los procesos sociales, que es expresión de los más altos valores de respeto a la dignidad del hombre, el derecho pleno a la vida, sean cuales fueran sus condiciones físicas, psíquicas y sociales; en cuyo centro de atención está brindar, todos los recursos necesarios para garantizar el desarrollo armónico e integral de la personalidad de los niños, adolescentes y jóvenes. Esto constituyó una de las motivaciones esenciales para la realización del estudio acerca de cómo se aprende, cómo se aprenden los conceptos y cómo se relacionan y cómo ha sido esta mirada desde diferentes concepciones y sus implicaciones en la formación de los docentes para la atención educativa a la diversidad de niños y aprendizajes en sus aulas.

Estos estudios sugieren, entre otros aspectos la necesidad creciente de continuar con el desarrollo de las ciencias pedagógicas, en la búsqueda de indicadores que orienten al maestro en los procesos de evaluación e intervención, de aquellas variables que influyen en el aprendizaje de sus propios alumnos para lograr el desarrollo y la formación de la personalidad desde el proceso pedagógico.

Es precisamente, en dar una respuesta a dicha problemática, que se han identificado en los planteamientos del enfoque histórico - cultural (Vigotsky, 1989 y 
seguidores Leontiev, Galperin, Talízina, y entre otros estudiosos, entre ellos cubanos como Siveiro, Morenza, Silvestre, Bell y otros) y de algunas posiciones de la Psicología Cognitiva con orientación dialéctico - materialista (Klix, F., Hoffmann), así como de las investigaciones de la autora de este artículo y algunos de sus discípulos (Matos Z. , Saavedra H.) que desde el año 2000 a la actualidad, han realizado aportaciones teóricas, metodológicas y prácticas.

Estas aportaciones han influido en el desarrollo histórico social de las ciencias psicológicas, pedagógicas y didácticas, en particular, en la concepción del diagnóstico del aprendizaje de los conceptos de los conceptos científicos y en la preparación de los docentes, por las posibilidades que presentan a la hora de describir y explicar el proceso del conocimiento humano, y de esclarecer las leyes del desarrollo de los niños con y sin discapacidad alguna, así como consideraciones para la dirección del proceso de enseñanza aprendizaje de los conceptos científicos desde determinadas asignaturas por el docente.

El diagnóstico es considerado una función inherente a la actividad pedagógica, constituye un principio pedagógico, es parte del quehacer de la escuela, de la actividad en el aula, mediada por el docente, el alumno y sus compañeros de aula, donde se significa la interacción entre los momentos de evaluación e intervención dirigida a objetivos determinados. Este se asume como un proceso de conocimiento de posibilidades, con un carácter explicativo y orientado a la intervención psicopedagógica fundamentalmente.

Los conceptos son considerados la piedra angular del conocimiento, reflejan la experiencia humana en su medio y se constituyen en uno de los elementos esenciales del aprendizaje, por esta razón es de interés comprender el estado y las posibilidades de desarrollo de las estructuras de los conocimientos. Estas son las formas en que se depositan los conocimientos en la memoria semántica, en ella no sólo se encuentran los conceptos y sus relaciones con otros conceptos, sino además las formas de operar con ese conocimiento. (Elosúa, 1986; Morenza, 1998, Hernández, 2003; 2011)

Estos estudios han demostrado en sus resultados científicos las posibilidades de determinar la diversidad en el proceso de asimilación de la experiencia social e individual. Además constituyen un índice del desarrollo de las capacidades intelectuales de un niño, en la medida en que se observan las posibilidades del sistema cognitivo de utilizar los 
conceptos y sus múltiples relaciones en la solución de nuevas tareas, pero también de la calidad de la interacción social (del plano interpsicológico al intrapsicológico).

De lo anterior se deduce, cuánto pueden expresar los conceptos acerca de la historia de aprendizaje de un niño, su desarrollo actual y potencial. La cantidad y calidad de conceptos de los que se apropia un niño, de cómo manipula esas bases de conocimientos y su capacidad de deducir el principio de solución de una tarea a partir de reconocer la pertenencia de un concepto a una clase y llevar a cabo un proceso de inclusión, es un indicador del potencial de aprendizaje al poder operar con conocimientos generalizados.

La investigación acerca de los conceptos, las estructuras del conocimiento y sus posibilidades para el diagnóstico del desarrollo y el aprendizaje de los alumnos de la educación básica primaria y de aquellos que asisten a la educación especial se ha implementado en Cuba desde finales de la década de los años '80 del pasado siglo en colaboración con el Centro de Neurociencias, la Facultad de Psicología de la Universidad de la Habana, y de Ciencias Pedagógicas de La Habana (Morenza y otros, 1990; Hernández, 2003; 2011; Matos, 2014; Saavedra, 2015).

Estos estudios han abordado la estructuración categorial, las categorías naturales en una población de niños de edad escolar, de ejemplares no pertinentes en la categorización, el diagnóstico de las estructuras de conocimiento en niños con desviaciones en el desarrollo mediante métodos diagnósticos como "la prueba de Conceptos con tareas de inclusión y exclusión", una concepción teórico metodológica para el diagnóstico del aprendizaje de los conceptos en niños con y sin discapacidad, análisis metodológico para la dirección del proceso de enseñanza aprendizaje de los conceptos naturales y sociales.

Los estudios expuestos revelan la preocupación y profundización en el contexto de la comunidad científica cubana acerca del diagnóstico del aprendizaje de los conceptos desde un enfoque psicológico, psicofisiológico, pedagógico y didáctico. A estos últimos tributan en lo fundamental los resultados de esta investigación, la cual considera que las estructuras del conocimiento son resultados de un aprendizaje científicamente dirigido.

En este sentido, la autora del presente estudio ha apreciado diversos comportamientos que, denotan limitaciones en el aprendizaje de los conceptos y las relaciones de 
significado en niños de la edad escolar que asisten a escuelas primaras y especiales, así como la ausencia de acciones de evaluación e intervención por maestros y especialistas de escuelas y centros de diagnóstico y orientación.

Estos elementos antes presentados se constatan además como preocupaciones en investigadores, especialistas y docentes con respecto al aprendizaje de los conceptos en los niños de la edad escolar, las que se manifiestan con carencias de palabras que refieran las acciones, las características de estas y sus relaciones entre ellas, evidencia un limitado vocabulario, así como la presencia del "verbalismo", todos los cuales reflejan cómo ha sido el proceso de internalización de la experiencia social e individual.

En las exploraciones en la práctica se observa la presencia de una concepción didáctica apoyada en la percepción de los objetos y fenómenos del medio, en el uso del lenguaje, consideración hacia los mediadores de carácter semánticos, al no sistematizarse acciones relativas a la evaluación e intervención de los conceptos, sus características generales, específicas, relaciones entre estas, de modo que permitan atender esas necesidades y potencialidades en los niños según su situación de desarrollo.

Los estudios científicos en poblaciones de alumnos con diversos diagnósticos clínico psicopedagógicos en el área sensorial e intelectual revelan necesidad de profundizar en las características del aprendizaje conceptual, como una arista del desarrollo infantil, de elevada significación para la atención educativa a la diversidad desde el proceso de enseñanza aprendizaje, se evidencian un tratamiento desde el punto de vista teórico y metodológico aún insuficiente.

Estos argumentos son precisamente los que han sido considerados para expresar en la contemporaneidad una respuesta desde posiciones pedagógicas y didácticas desde lo histórico - cultural con lo mejor de las contribuciones cognitivistas, las que han permitido esclarecer y aportar a la dirección del proceso de enseñanza - aprendizaje, nuevos indicadores para conocer y potenciar el desarrollo infantil desde el aprendizaje conceptual.

\section{Algunas consideraciones teóricas como punto de partida}

Algunas consideraciones teóricas como punto de partida de este tema se ubican en la escuela histórico - cultural, y en los planteamientos de la corriente cognitivista de orientación dialéctico materialista, así como en las reflexiones de la pedagogía y didáctica 
que se formulan en las investigaciones cubanas. A partir de la integración de estas ideas se aproxima a un recurso metodológico para el diagnóstico del aprendizaje de los conceptos, o sea, evaluar e intervenir acorde a las peculiaridades de los alumnos.

"... el concepto no es una formación aislada, osificada, que no sufre cambios, sino una parte activa del proceso intelectual, comprometida constantemente en servir a la comunicación, a la comprensión y a la solución de problemas" (Vigostki, 1968: 34). Con esta frase con profundas raíces histórico - cultural, se abordan el aprendizaje de los conceptos, la necesidad de su diagnóstico y la valoración de los docentes como un indicador para la dirección del proceso de enseñanza aprendizaje.

Desde esta visión, se extrae la significación que tienen los procesos psicológicos en la formación de los conceptos y su implicación en la actividad de comunicación, resulta de gran valor teórico para abordar el aprendizaje de los conceptos como una necesidad en la dirección de la actividad pedagógica del docente, ya que es punto de partida para comprender las regularidades que en dichos procesos presentan sus alumnos. Explorar estos procesos, conocer cómo funciona y por qué, es tarea principal del docente para organizar y dirigir el proceso de enseñanza- aprendizaje.

Para Vigotsky la formación de un concepto es un proceso que tiene lugar desde los preconceptos, los pseudoconceptos y el complejo. Precisamente, en cada una de estos momentos se expresa la experiencia humana, social e individual, la cual expresa niveles de estructuración acorde a la mediación; se reconocen los orígenes en esas interacciones, expresan los diversos vínculos entre el sujeto y los objetos, fenómenos, es reflejo de pensamiento coherente y objetivo según la unidad y diversidad en la realidad circundante.

Los estudios desde los avances de la neurociencias, la psicología cognitiva y la pedagogía y la didáctica en las últimas décadas hasta la actualidad han permitido penetrar en la comprensión de aquellas relaciones a las cuales se hace referencia, las que se establecen entre los objetos y fenómenos, por sus atributos, pero también de causa efecto, de locación, de receptor y portador, y que están estrechamente, relacionadas con las experiencias y vivencias del niño, aspecto este de elevada significación en la atención educativa a la diversidad acorde a los aprendizajes adquiridos y las nuevas formas de aprender a considerar desde el proceso de enseñanza. 
En esta fase al igual que la anterior se destacan cinco tipos de complejos, los que se suceden en esta etapa de desarrollo, por lo que no se dan de forma pura: asociativos, colecciones, en cadenas, difuso y los pseudoconceptos. Con respecto a estos se plantea que"... sirven como eslabón de enlace entre el pensamiento en conceptos y el pensamiento en complejos y posee una naturaleza dual, ya que el complejo también es portador de la semilla que hará germinar el concepto“"(Vigostki, 1968: 43).

En esta etapa juega un papel esencial la palabra del adulto, con respecto a ello se precisa que "el intercambio verbal con los adultos se convierte así en un poderoso factor en el desarrollo de los conceptos del niño" (Ibídem) Este punto de vista revela la importancia y el papel que desempeña el adulto en el desarrollo de los niños y en la formación de los conceptos, el cual actúa como mediador en las relaciones que ellos establecen con el medio mediante la comunicación, pues le transmite experiencias y cultura, o sea, aprendizajes que evolucionan en correspondencia con el nivel de desarrollo intelectual que hayan alcanzado. Aunque esta transmisión no ocurre de modo exacto a como el adulto piensa, permite que se activen en el niño procesos y formas de pensamiento conceptual, aspecto este muy importante para reconocer que mientras mayor preparación posea el maestro para dirigir el aprendizaje conceptual en los escolares, mejores condiciones estarán creando para la apropiación de estos.

En la etapa siguiente denominada por el propio autor como "conceptos potenciales" aparecen operaciones más complejas del pensamiento como la abstracción de tipo empírica, la que permite se realice una elección basada en determinados atributos comunes, aunque estas son inestables, en la edad infantil. También profundiza en el desarrollo de conceptos científicos, aquellos que se enseñan en la escuela y esboza que para su aprendizaje "requieren de la evolución de las funciones intelectuales siguientes: la atención deliberada, la memoria lógica, la abstracción, la comparación...", los que, como procesos psíquicos, no pueden ser dominados a través del aprendizaje aislado.

El análisis de tales preceptos formulados por Vigostki revela la necesidad de profundizar en el diagnóstico de los escolares, para no transmitirles aprendizajes acabados, sino conducirlos desde la búsqueda intelectual, la reflexión y el debate hacia el desarrollo gradual de habilidades propias del pensamiento científico que les permitan identificar lo esencial y las múltiples relaciones que se establecen entre los componentes 
del mundo natural y social, atendiendo a las particularidades y características propias de los escolares que les posibiliten alcanzar paulatinamente este momento del desarrollo.

En relación con lo anterior Vigostki señala que “... la instrucción es una de las fuentes principales de los conceptos infantiles y también una fuerza poderosa en la dirección del desarrollo de estos; determina el destino de la evolución mental completa (Vigostki, 1968: 54). Sentar las bases y crear las condiciones para el aprendizaje de los conceptos es misión de la escuela desde la organización del currículo escolar en relación estrecha con los momentos del desarrollo de los escolares, atendiendo a las potencialidades que presentan en su desarrollo a partir de sus necesidades y vivencias.

Desde este enfoque los conceptos son considerados como el reflejo subjetivo del mundo objetivo, su reflejo en la conciencia del hombre de la esencia de los objetos o clases de objetos, de los nexos esenciales acorde a la realidad que experimenta, vivencia, comprende, generaliza las características generales y específicas de los objetos o fenómenos reflejados en el pensamiento del niño.

El análisis que se realiza permite realizar la siguiente consideración, de las definiciones anteriores, se infiere la necesaria relación que ha de establecer el hombre con su medio desde la actividad y la comunicación, proceso este que se da en todos los individuos acorde a las condiciones de vida y de desarrollo vividos y es durante este proceso que se da el aprendizaje. Rico (2001: 13) lo significa como "el proceso de apropiación por el niño de la cultura, bajo condiciones de orientación e interacción social. Hacer suya esa cultura, requiere de un proceso activo, reflexivo, regulado, mediante el cual aprende de forma gradual, acerca de los objetos, procedimientos, las formas de actuar, las formas de interacción social, de pensar, del contexto histórico social en el que se desarrolla y de cuyo proceso dependerá su propio desarrollo“.

A partir de esta definición se revelan los rasgos esenciales del aprendizaje, como proceso que ocurre durante toda la vida, en él cristaliza continuamente la dialéctica entre lo histórico social y lo individual personal, posee un carácter tanto intelectual como emocional e individual que requiere participación, colaboración e interacción, multidimensional por sus contenidos, procesos y condiciones, posee diversos contenidos, ocurre durante la actividad, la comunicación y como resultado de ellas. 
En Cuba el aprendizaje de los conceptos ha sido un tema investigado por algunos autores desde el pasado siglo, (Morenza, 1990; Torres, 2000; Hernández, 2003; 2011), a partir de una concepción dialéctico - materialista del conocimiento, formularon interesantes ideas, específicamente, acerca del rendimiento intelectual desde una perspectiva cognitivista, las estructuras del conocimiento, las categorías y el aprendizaje de las relaciones semánticas que se establecen entre los conceptos de diferentes tipos. Estos autores, consideran la memoria como producto del procesamiento de la información, por lo que el tipo de representación conceptual está determinado por la cualidad de los procesos cognoscitivos que constituyen la base de su información.

La memoria es considerada un sistema funcional que almacena los resultados de los procesos de procesamiento de la información y los conserva listos para los siguientes procesos cognoscitivos. Estos resultados se ordenan acorde a su complejidad, asumen determinadas estructuras cognitivas, que multiplican las posibilidades de utilización simultánea, así como que se disminuye el gasto cognitivo de un sujeto, elementos estos que varían de un sujeto a otro. Precisamente, estos estudios acerca de la memoria revelan como en esta tiene lugar una compleja actividad dinámica cognoscitiva del sujeto en su relación con el medio y el procesamiento de la información que se manifiesta en diversas formas de percepción y de comportamiento en cada sujeto, y se requieren de recursos para profundizar, conocer sus vivencia, experiencias, la internalización de estas, su construcción en nuevos conocimientos, su comprensión, percepción y en el comportamiento personal, social.

En estas estructuras cognitivas se pueden apreciar diferentes tipos de redes, que circulan entre sí los conocimientos. Los estudios de estos autores revelan las carencias en los procesos de la actividad cognoscitiva de los alumnos los cuales deben ser considerados por los maestros en la dirección del proceso de enseñanza - aprendizaje. Donde además un elemento esencial lo constituye las relaciones necesarias: entre el componente motivacional con la cognición y de esta con la personalidad como partes que interactúan en el sistema de regulación del comportamiento humano.

El estudio de la memoria de larga duración o semántica implica comprender las tres funciones básicas y los necesario dos principios de almacenamiento y representación de la información; la clasificación que tiene lugar en los procesos de reconocimiento, la 
reproducción que se encuentra en la reactivación de estructuras complejas tras la excitación de zonas locales y la producción que se reconoce en los procesos de recombinación de contenidos en la memoria mediante inferencias o procesos lógicos.

Los principios de almacenamiento se expresan en la relación entre estados y procedimientos, es decir, entre estructuras estacionarias o estados y los procesos operativos o procedimientos; ambos se almacenan. El almacenamiento estacionario parte de la identificación de la correspondiente relación y de la activación de otras redes semánticas, lo que está influenciado por la complejidad del acto de identificar. "En esta forma de almacenamiento se configuran unidades conceptuales que representan un tipo de acontecimiento mediante relaciones semánticas" (Klix, 1987: 47).

Las relaciones semánticas son asociaciones entre las representaciones conceptuales en la memoria, surgidas como resultado de procesos cognitivos, los cuales reflejan la objetividad y especificidad de las relaciones reales del sujeto en su interacción con el medio y de la construcción individual. Estas pueden constituir indicadores, cuyas áreas deben ser atendidas en el proceso pedagógico. (Hernández, 2003; 2011).

Estos argumentos puntualizan la importancia de los procesos intelectuales en la formación de los conceptos, aspecto de interés para la investigación, pues en ellos se analiza la memoria como proceso fundamental donde se almacenan, estructuran y median en las respuestas ante el aprendizaje en forma de establecimiento de las diversas relaciones que se dan entre los objetos, fenómenos del mundo natural y social.

Otro principio de almacenamiento es aquel que se refiere al almacenamiento procesal, así las relaciones semánticas no son almacenadas como unidades en la memoria, sino como "prescripciones operativas" que permiten llegar a probar la presencia o no de una determinada relación (Klix, 1987). Este almacenamiento procesal implica dos premisas: debe tener lugar un procedimiento que posibilite probar o no la existencia de relaciones e implica un procedimiento de comparación de características.

Se destacan a partir de estas ideas tres formas de representación del medio en la memoria humana: los resultados de análisis sensorial de las estimulaciones, la representación lexical, en la que se almacenan unidades lingüísticas y las representaciones conceptuales. Estas últimas son aquellas donde se almacenan los resultados de los procesos conceptuales de abstracción; es decir, que independientemente de los efectos 
sensoriales concretos, se representan significados conocidos y relaciones semánticas, se almacena el conocimiento sobre las propiedades y relaciones de la realidad en una forma simbólica abstracta.

Las formas de representación del medio antes mencionadas, permiten comprender la manera en que puede el alumno del nivel educativo primario representarse el medio que le rodea y a su vez presupone la necesidad de la estimulación de los procesos que participan en el aprendizaje conceptual; entre otras, las sensaciones, las representaciones, la imaginación, a partir del diseño de variadas actividades por el docente que potencien esta estimulación, desde la clase.

"...en el curso de su vida, el hombre se apropia de las experiencias de las generaciones anteriores en la medida en que aprende a dominar significados. El significado deviene así la forma en la que el individuo se apropia de la experiencia humana reflejada y generalizada" (Leontiev, 1966: 181).

Estas ideas parten de la suposición de que dos clases de ordenamiento determinan la representación conceptual del mundo: interconceptual e intraconceptual (Klix, 1976; Hoffman, 1987).

"El ordenamiento interconceptual surge en la actividad propia y la observada, el cual conecta las acciones con las actuantes, con motivos y metas, con instrumentos y objetivos. Con él los conceptos están conectados mediante relaciones semánticas de diferentes tipos". (citado por Hoffman, 1987: 64.).

Aspecto este de un alto valor didáctico y metodológico para el diagnóstico en la dirección del proceso de enseñanza aprendizaje.

Estudios realizados por estos investigadores revelan que las relaciones interconceptuales influyen positivamente en la reproducción como función básica de la memoria, ya que determinan cada vez en mayor medida su secuencia, así como el aumento creciente del establecimiento de relaciones entre conceptos, siempre y cuando estas unidades estructurales estén determinadas por la integración de los elementos de la acción. Aquellos conceptos que refieren acción constituyen la génesis de la formación de las estructuras conceptuales, el niño aprende en y desde la actividad, la vivencia, la unidad entre los procesos cognitivos y afectivos desde la acción permiten una mejor comprensión del contenido de los objetos y fenómenos del medio. Aspecto este que aparece 
insuficientemente tratado en la literatura revisada desde el punto de vista didáctico y metodológico.

El otro aporte al diagnóstico del aprendizaje de los conceptos de esta teoría ha sido el reconocer que

"El ordenamiento intraconceptual es aquel ordenamiento clasificatorio que agrupa en clases los objetos sobre la base de sus características generales y específicas, de modo tal que en caso de cambios en las actitudes, patrones de conducta comparables, están ligados a clases conceptuales, según el caso" (Hoffman, 1987: 64).

En este los conceptos se encuentran unidos por relaciones de propiedades, de pertenencia, coordinación, supraordenación, nivel básico y subordinación.

A partir de estos estudios se fundamenta el papel que tiene la actividad del hombre en el intercambio de información con el medio, en el proceso del conocimiento; el hombre aprende mediante la actividad. El reflejo de la realidad objetiva tiene lugar inicialmente por las relaciones que se desarrollan a partir de su actividad propia. Mediante el intercambio continuo y sistemático con su medio (actividad y comunicación) en su vida familiar, escolar y social se reflejan con mayor plenitud las relaciones de tipo interconceptual, las que van ganando en estructuración.

Estas ideas permiten comprender el papel de las relaciones humanas, el intercambio que se produce con el medio en el aprendizaje de los conceptos, aspecto este a tener presente durante el proceso de enseñanza-aprendizaje, en el que el docente tiene la responsabilidad de explorar el estado de las estructuras de conocimiento, las experiencias y vivencias, los conceptos aprendidos para la construcción de otros nuevos. (Matos, 2014)

Otras investigaciones realizadas por Hernández (2003; 2011), Matos (2014), Saavedra (2015), específicamente, en escolares con necesidades educativas especiales dirigidas al aprendizaje de los conceptos revelan un conjunto de exigencias, las que son retomadas en este estudio para el trabajo propiamente didáctico del problema, precisamente, porque permiten desde un enfoque histórico - cultural, concebir el proceso de enseñanza- aprendizaje de los conceptos naturales y sociales como base para la comprensión de los conceptos científicos. 
El diagnóstico del aprendizaje de los conceptos requiere de las siguientes exigencias como referentes teóricos para la solución del problema pedagógico planteado:

- Determinación del nivel de ordenamiento del tipo de concepto, caracterización del conocimiento previo a partir del nivel interconceptual: relaciones semánticas de lugar, finalidad, objeto, instrumento, causa-efecto, cualidad.

- Identificación de relaciones intraconceptuales propias del proceso de aprendizaje a partir del tipo de ordenamiento dado.

- Determinación de la integración de los conceptos y sus relaciones desde situaciones complejas.

- Verificación del estado de aprendizaje conceptual a través de procesos lógicos del pensamiento como análisis y la síntesis, la comparación, en el que se privilegien las diferencias y la utilización de acciones de inclusión y de exclusión.

- La concientización de los resultados del aprendizaje de los conceptos y sus relaciones por el escolar mediante la utilización de otros recursos y vías

La determinación del nivel de ordenamiento del tipo de concepto implica dos maneras; una requiere de la reflexión por parte del docente del tipo de concepto que trabajará, (a qué nivel de ordenamiento conceptual pertenece) para establecer así las exigencias y expectativas en las respuestas de los alumnos acerca del aprendizaje logrado, lo que le permitirá conducir acertadamente el proceso, en el sentido de que el concepto debe estar comprendido en lo que el alumno debe conocer por sus experiencias, vivencias, el nivel escolar logrado a partir de ellos habrá de aprender lo nuevo.

La otra idea se concretará por las características de las respuestas que el alumno emita al planteársele uno u otro tipo de concepto, qué relaciones semánticas utiliza en torno al concepto dado, lo que permitirá al docente identificar los contenidos y procesos lógicos del pensamiento, que él utiliza, ha desarrollado, así como determinar los recursos y ayudas necesarias para las nuevas tareas.

La caracterización del conocimiento previo a partir del nivel interconceptual: relaciones semánticas de lugar, finalidad, objeto, instrumento, causa-efecto, cualidad, entre otras, indica que la caracterización acerca del conocimiento previo que sobre los objetos y fenómenos que poseen los alumnos debe realizarse mediante el reconocimiento 
del concepto que se establece a partir de uno que refiera acción, el cual da origen a la formación de diversas relaciones semánticas con orientaciones diferentes.

Se obtiene aquí un indicador significativo para el análisis del aprendizaje de los conceptos asimilados: la flexibilidad que se refiere a la posibilidad de acceder a campos semánticos diferentes, la diversidad de relaciones de significados variadas, las posibilidades de relacionarlas a su vez con diferentes naturalezas de los conceptos. Este resultado permite valorar a los conceptos que refieren acción como aquellos de amplias posibilidades para ampliar las estructuras de conocimientos en los alumnos, al ser estos la génesis de la formación de nuevos conceptos y relaciones entre estos.

La identificación de relaciones intraconceptuales propias del proceso de aprendizaje a partir del tipo de ordenamiento dado. Con esta exigencia se plantea un nivel estructural más complejo. Se refiere a la identificación de las características intraconceptuales generadas por un concepto dado, lo que incluye necesariamente un análisis vertical del aprendizaje del concepto desde sus características generales y las específicas y viceversa. Se puede así detectar no solo la extensión del concepto, sino la calidad con la que se subordinan a un concepto supraordenado, de ahí el análisis acerca de la representatividad de los conceptos que utiliza el alumno.

La representatividad de un concepto indica que este constituye un reflejo objetivo de la realidad, vivenciada, en cuanto a su significado social y al sentido personalizado para el sujeto, el cual ha de explorarse mediante los argumentos del alumno en la respuesta dada; a través de los procedimientos de comparación de palabras e imágenes ilustradas se puede identificar la representatividad y amplitud de los conceptos, su extensión en los dominios por el alumno, todo lo cual habla de la experiencia, del aprendizaje alcanzado y sus potencialidades.

La determinación de la integración de los conceptos y sus relaciones desde situaciones complejas, expresión de las interrelaciones con las anteriores, estas se complementan e integran de una forma dialéctica, en el proceso de conocimiento del alumno en su relación con el medio social. En dicho proceso de conocimiento se integran en una misma configuración conceptual tanto las relaciones inter como intraconceptuales en el contexto de una situación de aprendizaje, de ahí su carácter complejo. 
La verificación del estado de aprendizaje conceptual a través de procesos lógicos del pensamiento como análisis y la síntesis, la comparación, en el que se privilegien las diferencias y la utilización de acciones de inclusión y de exclusión. En esta exigencia, un papel esencial lo tienen las tareas de aprendizaje con acciones de incluir y excluir que desde la planificación de la clase y en correspondencia con el estado de los procesos cognitivos y afectivos de los alumnos elabore el docente, en las que se revele que durante el proceso de enseñanza-aprendizaje, se apliquen a nuevas situaciones el concepto o los conceptos aprendidos a partir de las relaciones que se dan. Estos elementos deben ser previamente concebidos desde la clase por el docente para la dirección de este proceso. De ahí el valor del análisis metodológico de la unidad de contenidos a trabajar a partir del diagnóstico del aprendizaje de los conceptos en los alumnos.

La concientización de los resultados del aprendizaje de los conceptos y sus relaciones por el alumno mediante la utilización de otros recursos y vías. La utilización de los recursos metodológicos propios de esa actividad como la elaboración de esquemas, listas de palabras, elaboración de mapas conceptuales, tareas de reconocimiento conceptual, entre otros, es valiosa para la retroalimentación de los resultados y la demostración del desarrollo alcanzado tanto en el trabajo grupal como individual.

Las exigencias metodológicas antes referidas se constituyen en elementos fundamentales a considerar en el proceso de enseñanza- aprendizaje de los conceptos y constituyen puntos de vistas esenciales a tener presente como contenido de este proceso en la preparación metodológica, las cuales se integran y forman un sistema en el proceso de evaluación e intervención de las adquisiciones en el alumno.

Todo lo anteriormente planteado permite asumir los presupuestos teóricos en función de una concepción dialéctica del proceso de aprendizaje, el diagnóstico y de la dirección del proceso de enseñanza - aprendizaje por el docente. De ahí que esta concepción que se presenta se erige en los siguientes planteamientos: la relación del hombre con su medio se da mediada por la actividad, el papel de los mediadores anátomo - fisiológicos, instrumentales y sociales, el curso de las relaciones que establecen en el grupo social: la familia, escuela, puede aprender a realizar acciones con los objetos, manipularlos, comprender las relaciones y la unidad dialéctica entre los procesos de la actividad - la percepción - el lenguaje y el conocimiento. 


\section{Conclusiones}

El diagnóstico del aprendizaje de los conceptos en niños del nivel primario se establece en términos del diagnóstico del aprendizaje de las relaciones semánticas. Este diagnóstico fino tiene que penetrar en las relaciones interconceptual e intraconceptual para evaluar e intervenir las estructuras de los conceptos asimilados y los nuevos según sus peculiaridades, necesidades y potencialidades de la experiencia humana adquirida, las cuales deben ser consideradas en la dirección en el proceso de enseñanza - aprendizaje y comprendidas desde un modelo de asimilación.

La acción constituye la génesis de la formación de las estructuras conceptuales, por tanto, a través de ella se posibilita la activación y el desarrollo de las posibles, variadas, complejas y múltiples relaciones de significado que se dan entre los objetos y fenómenos que rodean al niño, en forma de reflejo de estos en su pensamiento, todo lo cual es resultado de la mediación, todo lo cual se da en las relaciones del niño con el medio socio - cultural.

Los estudios investigativos de la autora en este tema denotan relaciones entre las condiciones de vida y de educación y desarrollo con el sistema de relaciones que establece el niño con su medio, las cuales generan un estado de privación social y cultural (Vigotsky, 1989) de consecuencias para las posibilidades de un desarrollo armónico e integral de la personalidad de estos, y en particular para el aprendizaje de los conceptos desde la dirección del proceso de enseñanza aprendizaje por el docente, de ahí la necesidad de su diagnóstico.

\section{Referencias bibliográficas}

Elosua, J. (1986). Una revisión crítica de los modelos de memoria semántica. Revista de Psicología General y Aplicada, 41, 2, 265-287.

Klix F. (1990). Investigaciones acerca de los procesos de reconocimiento que tiene lugar entre los contenidos de la memoria. Revista de Psicología, 1, 90, 124-175.

Klix, F. (1987). Psicología de la Cognición. Consecuencias metodológicas, teóricas y prácticas. En Colectivo de autores, Psicología en el Socialismo, La Habana: Editorial Ciencias Sociales. 
Hernández C. (2013). El diagnóstico del aprendizaje de los conceptos en niños con necesidades educativas especiales. Un problema pedagógico. Revista Órbita Científica, 19, 72, s/p.

(2011). El diagnóstico del aprendizaje de los conceptos en niños con discapacidad visual. En C. Hernández, Desarrollo de las concepciones educativa en las personas con discapacidad visual. (pp. 53-118) La Habana, Cuba: Editorial Pueblo y Educación.

(2003) Concepción teórico metodológica para el diagnóstico del aprendizaje de los conceptos en niños con discapacidad visual. Tesis Doctoral: La Habana, Cuba.

Matos, Z y Hernández C. (2015), Consideraciones teóricas acerca del proceso de enseñanza aprendizaje. Principales tendencias en la enseñanza de los conceptos en escolares primarios. En A. Gamboa et al. Formación docente y Prácticas inclusivas. (pp. 151-166) Estado Sonora México: IFODES.

Matos, Z. (2014) Concepción metodológica para la dirección del proceso de enseñanza aprendizaje de los conceptos en escolares con Retardo en el Desarrollo Psíquico. Tesis doctoral: La Habana Cuba.

Morenza, L. (1990). La psicología cognitiva contemporánea y el desarrollo de las capacidades intelectuales. Congreso Internacional Pedagogía 90, La Habana Cuba: Palacio de las Convenciones

Rico, P. (2001). Hacia el perfeccionamiento de la escuela primaria. La Habana: Editorial Pueblo y Educación.

Saavedra, H. (2015), Concepción teórico metodológica para el aprendizaje de los conceptos científicos en escolares sordos, Tesis Doctoral: La Habana.

Vigotsky, L. (1989). Obras Completas. La Habana, Cuba: Editorial Pueblo y Educación.

Vigotsky, L. (1968). Pensamiento y lenguaje. La Habana: Editorial Revolucionaria. 